\title{
Real World Treatment Practices for Chronic Lymphocytic Leukemia in Japan: An Observational Database Research Study (CLIMBER-DBR)
}

\author{
Jun Takizawa, ${ }^{1}$ Koji Izutsu, ${ }^{2)}$ Hirokazu Nagai, ${ }^{3)}$ Kenjiro Fukase, ${ }^{4)}$ Maki Nakamura, ${ }^{4)}$ \\ Masahisa Jinushi, ${ }^{4)}$ Junji Suzumiya ${ }^{5)}$
}

There are limited real-world data on the treatment practices and healthcare resource utilization associated with chronic lymphocytic leukemia (CLL) in Japan. In this study (CLIMBER-DBR), we performed retrospective analyses of the Japanese Medical Data Vision database, and extracted data for 2562 patients with newly diagnosed CLL (CLL-1 cohort) and 930 patients receiving CLL treatment (CLL-2 cohort) registered between March 1, 2013 and February 28, 2018. The median follow-up in the CLL-1 cohort was 721 (quartile 1-3:363-1267) days and the median time to initial (first-line) treatment was 1331 (quartile 1-3: 189-not reached) days. In the CLL-2 cohort, the most frequently used regimens were fludarabine alone (17.7\%), cyclophosphamide alone (13.7\%), and bendamustine/rituximab (8.2\%). The median (quartile 1-3) times to second-line and thirdline treatments were 1066 (273-not reached) and 1795 (631-not reached) days, respectively. The CLIMBER-DBR was the first database research study to assess current treatment practices for CLL in Japan, where the treatment patterns were driven by the approval/reimbursement status of drugs in the study period. Our study provides an important benchmark for future studies of CLL in Japan.

Keywords: Chronic lymphocytic leukemia, Japan, real world, treatment patterns, resource utilization

\section{INTRODUCTION}

Chronic lymphocytic leukemia (CLL) is a relatively rare disease, with an annual age-adjusted incidence of approximately 0.2 cases per 100,000 people in Japan ${ }^{1}$ and an estimated 2000 patients nationwide. ${ }^{2}$ It is estimated that CLL accounts for $\sim 3 \%$ of all cases of malignant lymphomas in Japan,,$^{1,3}$ a much lower rate than that of $\sim 25 \%$ in Western countries. ${ }^{4}$

CLL has a long clinical course, and avoiding treatmentrelated deaths or adverse events (AEs) is an important aspect of its management. Therefore, it is important to start treatment by identifying the disease stage and activity. A recent review proposed an evolution of initial CLL therapy in Japan, ${ }^{5}$ extending the previous 2018 guidelines issued by the Japanese Society of Hematology (JSH), ${ }^{6,7}$ which are now mostly in line with the International Workshop on Chronic
Lymphocytic Leukemia guidelines. ${ }^{8}$ The JSH guidelines recommend a "watch and wait" approach initially for patients without active disease. For patients who do require treatment, the recommended regimens include ibrutinib, fludarabine + cyclophosphamide rituximab (FCR) and bendamustine + rituximab (BR).

In recent years, there have been marked changes to the treatment landscape in Japan, with the approval of rituximab for CLL in 2019 (previously approved for B-cell non-Hodgkin lymphoma in 2003), bendamustine in 2016, ibrutinib for relapsed/refractory CLL in 2016 and as first-line treatment in 2018, and venetoclax for relapsed/refractory CLL in $2019 .^{5}$ Overseas, other new treatments have been incorporated into the 2020 National Comprehensive Cancer Network Clinical Practice Guidelines, ${ }^{9}$ which recommend ibrutinib, acalabrutinib \pm obinutuzumab, or venetoclax + obinutuzumab as firstline treatment, of which only ibrutinib is approved in this set-

\footnotetext{
Received: September 11, 2020. Revised: February 18, 2021. Accepted: April 4, 2021. J-STAGE Advance Published: June 5, 2021

DOI: $10.3960 /$ jslrt.20044

${ }^{1}$ Department of Hematology, Endocrinology and Metabolism, Niigata University Faculty of Medicine, Niigata, Japan, ${ }^{2}$ Department of Hematology, National Cancer Center Hospital,

Tokyo, Japan, ${ }^{3}$ Department of Hematology, National Hospital Organization Nagoya Medical Center, Nagoya, Japan, ${ }^{4)}$ AstraZeneca K.K., Osaka, Japan, ${ }^{5)}$ Innovative Cancer Center, Shimane University Hospital, Izumo, Japan

Present affiliation: JS, Department of Hematology, Koga Community Hospital, Yaizu, Japan

Corresponding author: Jun Takizawa, MD, PhD, Department of Hematology, Endocrinology and Metabolism, Niigata University Faculty of Medicine, 1-757 Asahimachi-dori,

Chuo-ku, Niigata, 951-8510, Japan. E-mail: juntaki@med.niigata-u.ac.jp

Copyright (C) 2021 The Japanese Society for Lymphoreticular Tissue Research

(cc) BY-NC-SA This work is licensed under a Creative Commons Attribution-NonCommercial-ShareAlike 4.0 International License.
} 
ting in Japan.

Currently, there is limited evidence on how physicians in Japan treat CLL patients in real-world settings. In particular, it is unclear how long physicians wait until they prescribe first-line treatment for patients with newly diagnosed CLL. In addition, it remains largely obscure which treatment regimens are used or preferred by physicians in real-world practice, especially considering the recent changes to the treatment landscape in Japan. ${ }^{5}$ Information is also needed on the impact of CLL on healthcare resource utilization and expenditure.

Some insight into the real-world management of CLL came from a US study using a commercial healthcare insurance claims database of patients diagnosed between July 2012 and June 2015. ${ }^{10}$ In that study, BR was the most common first-line treatment regimen $(28.1 \%)$, with a median time to initial (first-line) treatment (TIT) of 3.3 months (versus 16.4 and 7.7 months for ibrutinib and rituximab, respectively). ${ }^{10}$ The authors also revealed a high cost of treating CLL, with mean monthly all-cause and CLL-related costs of $\$ 7943$ and $\$ 5185$, respectively, among patients prescribed systemic treatment.

Similar data are therefore needed in Japan in order to better understand the current clinical practices for CLL and its healthcare resource utilization. Accordingly, the CLIMBERDBR study was performed with the objectives of determining the characteristics, treatment patterns, and healthcare resource utilization of CLL in a real-world clinical setting in Japan using a secondary data source.

\section{MATERIALS AND METHODS}

\section{Ethics}

The MDV database (Medical Data Vision Co., Ltd., Tokyo, Japan), the database used in this study, comprises data collected under contract from the participating institutions. All data regarding personal information were anonymized by each institution and the database does not include the location of institution. Therefore, patient information cannot be retrieved from the database. This complies with the Act on the Protection of Personal Information in Japan. The contracts between MDV and the institutions permit this MDV database to be used for research purposes. The study was also approved by the Institutional Review Board of the Clinical Research Promotion Network-Japan (Osaka, Japan; approval number: 7625-NIS-8326-00; date: June 20, 2019).

\section{MDV database}

We obtained data from the MDV database, which records healthcare claims and diagnostic procedure combination information collected under consent from hospitals across Japan. As of February 2018, the database included data from 375 participating hospitals, including 187 cancer care hub hospitals, with over 10 million patients treated in inpatient and outpatient settings. All data are recorded in the MDV database anonymously. Diseases are classified using
International Classification of Diseases 10th Edition (ICD10) codes and treatments are recorded using Anatomical Therapeutic Chemical Classification (ATC) codes.

\section{Patients and definition of study cohorts}

We searched the MDV database for patients with an ICD10 diagnosis code of $\mathrm{C} 83$ for non-follicular lymphoma or C91 for lymphocytic leukemia, filtered with the Japanese claim code '8847359', with a study period of March 1, 2013 to February 28, 2018. As a "watch and wait" approach is often appropriate for patients without symptoms, we established two cohorts, CLL-1 and CLL-2, which were defined as follows: The CLL-1 cohort comprised patients with a diagnosis of CLL recorded during the study period who were aged $\geq 18$ years at diagnosis. The objectives of this cohort were to assess the characteristics of patients with newly diagnosed CLL and to determine the TIT from diagnosis. The CLL-2 cohort comprised patients with a diagnosis of CLL who received treatment for CLL during the study period at $\geq 18$ years of age. The objectives of this cohort were to assess the characteristics of patients who received antineoplastic agents as first-line or second-/third-line treatments, and to evaluate the characteristics of patients under treatment, and the times from the first prescription of antineoplastic treatment to first subsequent (second-line) treatment (TFST) and second subsequent (third-line) treatment (TSST). Both cohorts were also used to evaluate healthcare resource utilization and frequency of Richter's transformation. The index date was defined as the date of the first diagnosis of CLL in the CLL-1 cohort and as the date of first treatment in the CLL-2 cohort. Some overlap of the CLL-1 and CLL-2 cohorts was expected because of the possibility that some patients were first diagnosed and received antineoplastic treatment in the study period; patients who were first diagnosed but did not receive antineoplastic treatment in the study period were included in the CLL-1 cohort. Patients who were diagnosed before the study period but who received treatment during the study period were included in the CLL-2 cohort. We excluded patients aged $<18$ years at the index date, patients with an index date outside the study period, and patients who underwent stem cell transplantation before the index date.

\section{Measures and outcomes}

All data and clinical records, including diagnostic procedures, were retrospectively collected from the MDV database. Follow-up data were recorded for all patients until February 28, 2019, or until their last recorded visit or death. Baseline data included age, sex, body weight, and modified Charlson Comorbidity Index (mCCI). The mCCI was derived from the following items: myocardial infarction, congestive heart failure, peripheral vascular disease, cerebrovascular disease, dementia, chronic pulmonary disease, rheumatic disease, peptic ulcer disease, mild liver disease, diabetes without chronic complications, diabetes with chronic complications, hemiplegia or paraplegia, renal disease, moderate or severe liver disease, metastatic solid tumor, and acquired immunodeficiency syndrome/human immunodeficiency 
virus. mCCI included malignant neoplasm of the skin, but excluded hematological malignancies (lymphoma and leukemia). The prescription of antineoplastic agents and corticosteroids (when used in combination with antineoplastic drugs) was identified using the ATC codes L01 and H02AB, respectively. Antineoplastic medications were grouped by regimen and sequence for up to three lines of treatment per patient.

We assessed the frequencies of comorbidities and AEs that are commonly associated with CLL by considering the relevant treatments and medications as clinical events. For this, we retrieved information on in-hospital deaths, diagnosis of comorbidities, emergency hospitalization, and surgical/ diagnostic/medical procedures (including head and neck imaging, catheter ablation, and blood transfusion) from the MDV database. We also recorded the prescription of medications used to treat associated safety risks (e.g. systemic steroids that were not prescribed in combination with antineoplastic drugs, anti-infective agents, antiarrhythmic agents, antithrombotic agents, and urate-lowering agents). Due to the nature of the database, it was not possible to determine whether drugs were used for prophylactic or treatment purposes.

Healthcare resource utilization was assessed in terms of the number of hospitalizations, the duration of hospitalization, number of outpatient visits, number of emergency room visits, procedures, and medical examinations. The costs attributable to these events, in addition to the costs of medications and other costs, were retrieved from the healthcare claims data.

\section{Study outcomes}

The primary study outcome was to describe the characteristics of patients with newly diagnosed CLL (CLL-1 cohort) or patients receiving antineoplastic agents as first-line/subsequent treatment (CLL-2 cohort). Secondary outcomes included the characteristics of patients under treatment (CLL-2 cohort), TIT (CLL-1 cohort), TFST (CLL-2 cohort), TSST (CLL-2 cohort), clinical events (both cohorts), and medical resource utilization expressed in millions of Japanese yen (JPY) (both cohorts). The TIT was calculated as the time from the index date to the first prescribed antineoplastic treatment. TFST and TSST were calculated as the times from the first prescription of an antineoplastic treatment to the prescription of the second-line (TFST) or thirdline (TSST) treatments. As an exploratory endpoint, we determined the incidence of new diagnoses of diffuse large $\mathrm{B}$ cell lymphoma (DLBCL; ICD code C83.3) or Hodgkin lymphoma (HL; C81) as cases of Richter's transformation during the follow-up period. These outcomes were selected to provide insight into the treatment choices and disease progression in real-world settings in Japan, in addition to the healthcare resources utilized for the treatment of CLL and its associated comorbidities or AEs.

\section{Statistical analysis}

Data were analyzed using SAS version 9.3 or later (SAS
Inc., Cary, NC, USA). Patient characteristics, clinical outcomes, and resource utilization were assessed descriptively, and results are presented as the number (percent) of patients, mean \pm standard deviation, or median (quartile 1-quartile 3 [Q1-Q3]). Medications and treatment combinations were summarized descriptively for up to three lines of treatment per patient. The median (Q1-Q3) TIT, TFST, and TSST were calculated using the Kaplan-Meier method. As the diagnosis was classified as 'severe' in some patients, we performed sensitivity analyses using two modified cohorts: 'excomorbid' and 'LastDiag' cohorts. The 'excomorbid' cohort was established by excluding any patients with a diagnosis of hematological malignancy other than a CLL-related diagnosis. The 'LastDiag' cohort was limited to patients with a diagnosis of CLL that continued through to the end of the follow-up period. The sensitivity analyses using these modified cohorts involved assessment of patient characteristics and treatment sequences.

\section{RESULTS}

\section{Patients}

\section{CLL-1 cohort}

The CLL-1 cohort comprised 2562 patients who were first diagnosed with CLL during the study period (Figure 1). The median (Q1-Q3) follow-up period of these patients was 721 (363-1267) days. The mean age, body weight, and $\mathrm{mCCI}$ of patients in the CLL-1 cohort were 70.7 years, 56.5 $\mathrm{kg}$, and 2.2 , respectively, and $26.4 \%$ of patients were $<65$ years old (Table 1).

\section{CLL-2 cohort}

The CLL-2 cohort, defined as patients who received at least one CLL treatment during the study period, comprised 930 patients (Figure 1). Of these, 916 (98.4\%) overlapped with the CLL-1 cohort. Their median (range) follow-up period was $727.5(0-2169)$ days. The mean age was 70.1 years and $26.7 \%$ of patients were $<65$ years old at the index date. The mean weight and mCCI were $57.3 \mathrm{~kg}$ and 1.3 , respectively (Table 1). All 930 patients in the CLL-2 cohort were prescribed first-line treatment, 352 patients received two or more lines of treatment, and 160 received three or more lines of treatment during the study period.

\section{Time to first-line treatment in the CLL-1 cohort, treatment regimens, and times to second-/third-line treatments in the CLL-2 cohort}

The median TIT in the CLL-1 cohort was 1331 days (Q1-Q3: 189-not reached), which was calculated as the time from the first diagnosis to first treatment prescription or death (Figure 2a), the median TFST was 1066 days (Q1-Q3: 273not reached; Figure 2b), and the median TSST was 1795 days (Q1-Q3: 631-not reached; Figure 2c).

The treatment regimens used in $\geq 2 \%$ of patients for each line are listed in Table 2. As first-line treatment, the three 


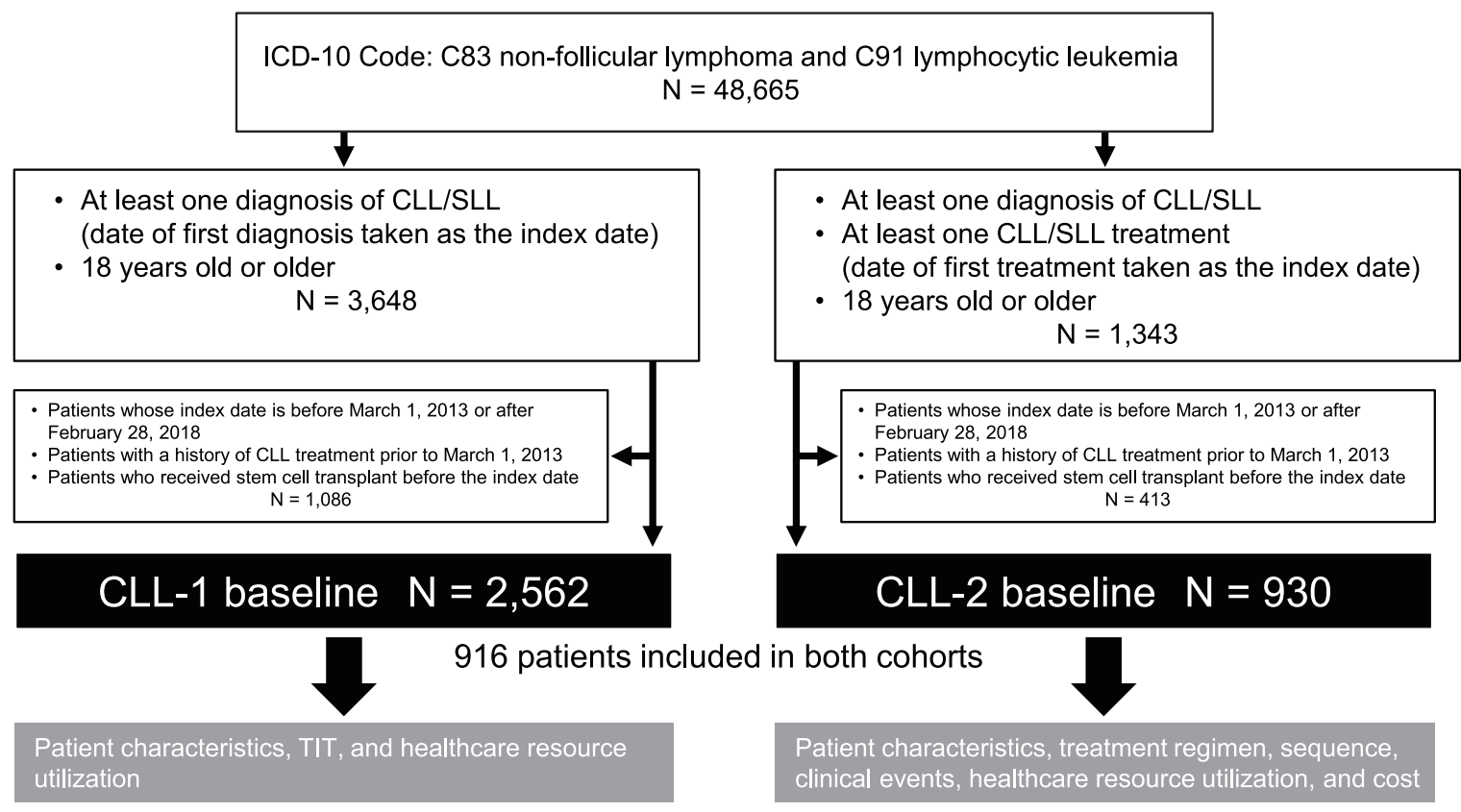

Fig. 1. Study population. The CLL-1 cohort comprised patients with a diagnosis of CLL recorded during the study period and the CLL-2 cohort comprised CLL patients who received treatment for CLL during the study period. Some patients were included in both cohorts

Table 1. Baseline characteristics of the CLL-1 and CLL-2 cohorts

\begin{tabular}{lcc}
\hline & CLL-1 & CLL-2 \\
& $\mathrm{N}=2562$ & $\mathrm{~N}=930$ \\
\hline Age, mean (SD) [range] & $70.7(12.3)$ & $70.1(11.7)$ \\
& {$[18-100]$} & {$[18-93]$} \\
Age $<65$ years, n (\%) & $677(26.4)$ & $248(26.7)$ \\
Male, $\mathrm{n}(\%)$ & $1481(57.8)$ & $587(63.1)$ \\
Weight (kg), mean (SD) [range] & $56.5(11.7)$ & $57.3(11.4)$ \\
& {$[23.7-122.8]$} & {$[29.1-112.0]$} \\
mCCI, mean (SD)* & $2.2(1.6)$ & $1.3(1.4)$ \\
& {$[0-7]$} & {$[0-8]$} \\
\hline
\end{tabular}

*Derived from the following items: myocardial infarction, congestive heart failure, peripheral vascular disease, cerebrovascular disease, dementia, chronic pulmonary disease, rheumatic disease, peptic ulcer disease, mild liver disease, diabetes without chronic complications, diabetes with chronic complications, hemiplegia or paraplegia, renal disease, moderate or severe liver disease, metastatic solid tumor, and acquired immunodeficiency syndrome/human immunodeficiency virus, excluding hematological malignancies (lymphoma and leukemia)

SD, standard deviation; mCCI, modified Charlson Comorbidity Index

most frequent regimens were fludarabine alone (17.7\%), cyclophosphamide alone (13.7\%), and BR (8.2\%). Ibrutinib was the most frequently prescribed second-line $(10.5 \%)$ or third-line (13.8\%) treatment. BR was the most frequent combination regimen used as second-line $(9.9 \%)$ or third-line $(5.6 \%)$ treatment. Among 538 patients who received firstline treatment prior to the approval of ibrutinib in May 2016, the first-line treatments prescribed to $\geq 5 \%$ of patients were fludarabine (19.3\%), cyclophosphamide (15.6\%), methotrexate $(6.5 \%)$, fludarabine/rituximab (5.8\%), BR (5.2\%), rituximab/ cyclophosphamide/doxorubicin/vincristine/prednisolone (R-CHOP; 5.2\%), and rituximab (5.0\%). Among 392 patients who received first-line treatment after this date, the most common regimens were fludarabine $(15.6 \%)$, BR $(12.2 \%)$, cyclophosphamide $(11.0 \%)$, methotrexate $(6.4 \%)$, ibrutinib $(6.1 \%)$, rituximab $(5.6 \%)$, bendamustine $(5.1 \%)$, and FCR $(5.1 \%)$. There were no notable differences in treatment patterns between cancer care hub hospitals and noncancer care hub hospitals (data not shown). The most common treatment sequences from first-line to second-line treatment, for patients who received fludarabine, cyclophosphamide, or BR as first-line therapy are listed in Table 3.

\section{Clinical events and associated safety risks in the CLL-2 cohort}

The most common clinical event recorded in the CLL-2 cohort based on the prescribed drugs or procedures was the prescription of systemic antibacterial drugs, which was recorded for $84.7 \%$ of patients (Table 4 ). Just over half of the patients (56.8\%) were prescribed intravenous antibiotics. Antithrombotic drugs were also prescribed to over half $(58.3 \%)$ of the patients (Table 4$)$. Other frequently recorded events were transfusion $(40.2 \%)$ and prescription of systemic antifungal drugs $(40.2 \%)$.

\section{Healthcare resource utilization and expenditure in the CLL-1 and CLL-2 cohorts}

Healthcare resource utilization in CLL is shown in Table 5. Half of the patients were hospitalized at least once, with a median $(\mathrm{Q} 1-\mathrm{Q} 3)$ of $2(1-3)$ times per patient and median (Q1-Q3) hospitalization of 29 (12-65) days. Overall, 19.4\% of patients were admitted to an emergency room for a median of 0.7 times per patient per year $(0.4-1.7)$. Most patients 
(a) Time to initial treatment: CLL-1

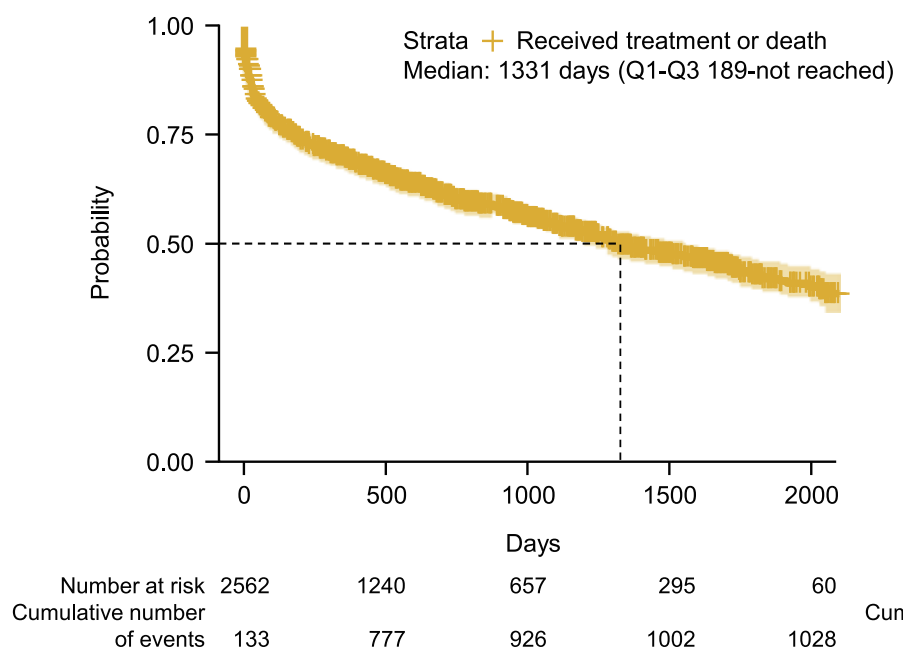

(b) Time to first subsequent treatment: CLL-2

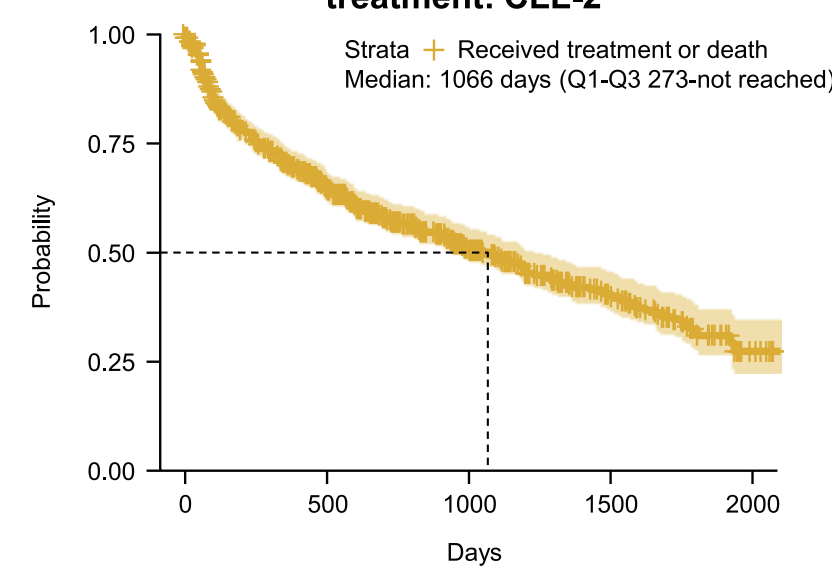

(C) Time to second subsequent treatment: CLL-2

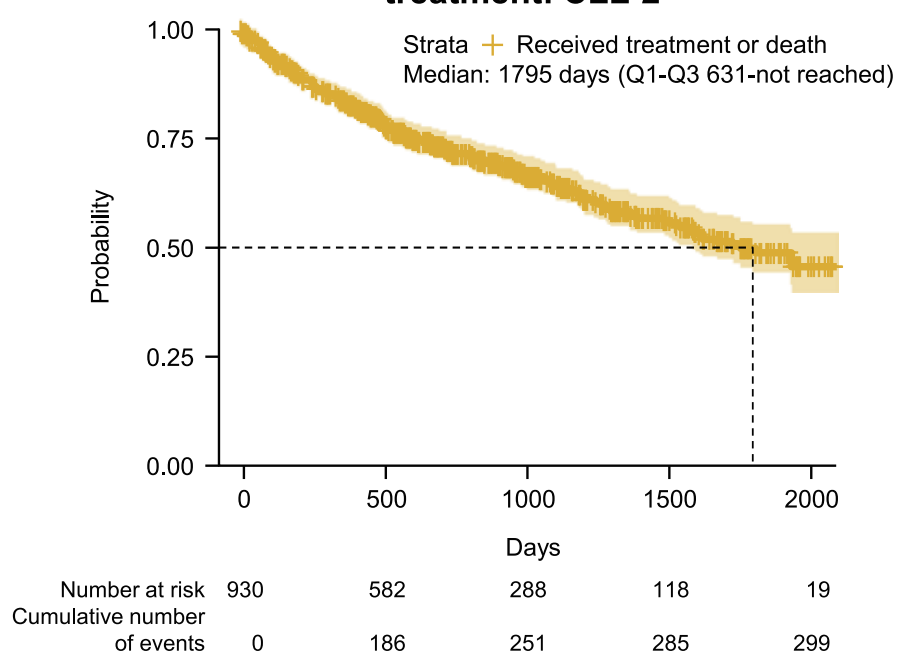

Fig. 2. (a) Time from the index date to the first prescribed antineoplastic treatment in the CLL-1 cohort (TIT). (b, $\boldsymbol{c}$ ) Times from the first prescription of an antineoplastic treatment to subsequent second-line $(\boldsymbol{b}$; TFST) and third-line treatments $(\boldsymbol{c}$; TSST) in the CLL-2 cohort

$(96.0 \%)$ attended outpatient visits at least once during the follow-up period, with a median of 10.7 (6.3-17.4) visits per patient per year. In terms of healthcare claims (Table 6), the median total cost was 0.94 million JPY per patient $(0.63$ million JPY per patient per year). Inpatient visits accounted for the bulk of the healthcare claims in the CLL-1 cohort $(1.72$ million JPY per patient).

Healthcare resource utilization and claims in the CLL-2 cohort are also shown in Tables 5 and 6 . As indicated, the frequency of patients requiring hospitalization at least once was $66.5 \%$ and the median number of outpatient visits per patient per year was $14.9(\mathrm{Q} 1-\mathrm{Q} 3: 10.2-21.1)$. The median total healthcare claim per patient was 4.24 million JPY (2.53 million JPY per patient per year).

\section{Richter's transformation}

The frequency of Richter's transformation was determined as an exploratory outcome in terms of new diagnosis of DLBCL or HL during the follow-up period. In the CLL-1 cohort, Richter's transformation accounted for 26 patients (1\%), including 22 with DLBCL and four with HL. In the CLL-2 cohort, transformation occurred in 20 patients $(2.2 \%)$, including 17 with DLBCL and three with HL.

\section{Sensitivity analysis}

The sensitivity analysis in the 'excomorbid' cohort (i.e., patients without hematological disorders other than CLL) revealed no appreciable differences in patient characteristics, treatment sequences, or commonly used regimens relative to the CLL-1 and CLL-2 cohorts. In this analysis, fludarabine 
Table 2. First-, second-, and third-line treatments used in $\geq 2 \%$ of patients by treatment line in the CLL-2 cohort

\begin{tabular}{|c|c|c|c|c|c|}
\hline \multicolumn{2}{|c|}{$\begin{array}{l}\text { First-line treatment } \\
\mathrm{N}=930\end{array}$} & \multicolumn{2}{|c|}{$\begin{array}{l}\text { Second-line treatment } \\
\mathrm{N}=352\end{array}$} & \multicolumn{2}{|c|}{$\begin{array}{l}\text { Third-line treatment } \\
\mathrm{N}=160\end{array}$} \\
\hline $\mathrm{F}$ & $165(17.7 \%)$ & Ibrutinib & $37(10.5 \%)$ & Ibrutinib & $22(13.8 \%)$ \\
\hline $\mathrm{C}$ & $127(13.7 \%)$ & $\mathrm{BR}$ & 35 (9.9\%) & $\mathrm{F}$ & $12(7.5 \%)$ \\
\hline $\mathrm{BR}$ & $76(8.2 \%)$ & B & $23(6.5 \%)$ & $\mathrm{BR}$ & $9(5.6 \%)$ \\
\hline Methotrexate & $60(6.5 \%)$ & $\mathrm{R}$ & $23(6.5 \%)$ & B & $8(5.0 \%)$ \\
\hline $\mathrm{R}$ & $49(5.3 \%)$ & $\mathrm{F}$ & $19(5.4 \%)$ & Etoposide & $8(5.0 \%)$ \\
\hline R-CHOP & $46(4.9 \%)$ & Ofatumumab & $18(5.1 \%)$ & $\mathrm{R}$ & $6(3.8 \%)$ \\
\hline FR & $42(4.5 \%)$ & $\mathrm{C}$ & $10(2.8 \%)$ & $\mathrm{C}$ & $4(2.5 \%)$ \\
\hline FCR & $35(3.8 \%)$ & FCR & $10(2.8 \%)$ & Ofatumumab & $4(2.5 \%)$ \\
\hline $\mathrm{FC}$ & $32(3.4 \%)$ & R-CHOP & $9(2.6 \%)$ & R-EPOCH & $4(2.5 \%)$ \\
\hline B & $27(2.9 \%)$ & Etoposide & $8(2.3 \%)$ & R-ICE & $4(2.5 \%)$ \\
\hline Ibrutinib & $24(2.6 \%)$ & & & & \\
\hline
\end{tabular}

F, fludarabine; C, cyclophosphamide; B, bendamustine; R, rituximab; CHOP, cyclophosphamide/doxorubicin/ vincristine/prednisolone; EPOCH, etoposide/prednisolone/vincristine/cyclophosphamide/doxorubicin; ICE, ifosfamide/carboplatin/etoposide

Table 3. Common first-line $\rightarrow$ second-line treatment sequences in the CLL-2 cohort

\begin{tabular}{llll}
\hline $\begin{array}{l}\text { First-line treatment } \\
\mathrm{N}=930\end{array}$ & & \multicolumn{2}{l}{$\begin{array}{l}\text { Subsequent second-line } \\
\text { treatment* }\end{array}$} \\
\hline Fludarabine & $165(17.7 \%)$ & Ibrutinib & $13(7.9 \%)$ \\
& & Ofatumumab & $10(6.1 \%)$ \\
& & Rituximab & $8(4.8 \%)$ \\
& & Other & $38(23.0 \%)$ \\
& & No treatment & $96(58.2 \%)$ \\
Cyclophosphamide & $127(13.7 \%)$ & Fludarabine & $10(7.9 \%)$ \\
& & Rituximab & $5(3.9 \%)$ \\
& & BR & $3(2.4 \%)$ \\
& & Other & $15(11.8 \%)$ \\
BR & & No treatment & $94(74.0 \%)$ \\
& & Ibrutinib & $7(9.2 \%)$ \\
& & Etoposide & $2(2.6 \%)$ \\
& & Other & $14(18.4 \%)$ \\
& & No treatment & $53(69.7 \%)$ \\
\hline
\end{tabular}

*Calculated as the percentage of patients who received the first-line treatment indicated in the first column

$\mathrm{BR}$, bendamustine + rituximab

was used in $24.0 \%$ of the overall patients, cyclophosphamide in $18.2 \%$, and BR in $11.2 \%$ (data not shown). The 'LastDiag' sensitivity analysis, in terms of patient characteristics, treatment sequences, and commonly used treatments, was also consistent with the 'excomorbid' cohort and the CLL-1 and CLL-2 cohorts.

\section{DISCUSSION}

We performed this study using the MDV database with several objectives that were designed to help understand the treatment practices for patients with CLL in Japan. The MDV database is suitable for assessing the study outcomes due to its large size, which enables researchers to assess relatively rare disorders in clinical practice. Indeed, the database registered data for 2562 patients with a new diagnosis of CLL; approximately 500 patients per year. This is substantial considering a prior study that estimated an agestandardized annual incidence of approximately 0.2 cases per 100,000 people. $^{1}$

The general patient characteristics (i.e. mean age, sex, body weight, and $\mathrm{mCCI}$ ) were comparable in both the CLL-1 and CLL-2 cohorts, and were similar to those of patients in other foreign studies of CLL. ${ }^{10-12}$

The JSH 2018 guidelines ${ }^{6,7}$ suggest several regimens, such as rituximab or ibrutinib, that were not necessarily approved for this indication at the time the guidelines were written. For example, rituximab was not approved for CLL until 2019; before that, it was approved for the treatment of B-cell non-Hodgkin lymphoma in 2003. Bendamustine was approved for CLL in 2016, whereas ibrutinib was approved for relapsed/refractory CLL in 2016 and as first-line treatment in 2018..$^{5}$ Our study suggests that Japanese physicians often prescribed cyclophosphamide- or fludarabine-based regimens as the main first-line treatments for CLL before the approval of novel targeted drugs such as ibrutinib. The most common second-line treatments were ibrutinib, BR, bendamustine, rituximab, and fludarabine. These treatment patterns are similar to those reported in another Japanese study, ${ }^{13}$ but differ somewhat from those in other countries, especially the US, ${ }^{10,12,14}$ where BR and ibrutinib are predominantly used. These differences likely reflect the earlier approval of the newer CLL treatments in the US and differences in reimbursement status. Some Japanese physicians may have preferred monotherapies and oral regimens associated with fewer side effects and lower costs when treating CLL patients, and took into account multiple patient factors in the treatment decisions such as age. They may also have considered social or environmental factors like how far the patient lives from the hospital/clinic, social support, and the patient's economic situation. Ibrutinib was used as a firstline treatment in some patients in this study, which is unexpected considering it was not approved for first-line use until 
Table 4. Clinical events and associated safety risks in the CLL-2 cohort

\begin{tabular}{llc}
\hline Event* & Associated safety risk & Total \\
& & $\mathrm{N}=930$ \\
\hline Prescription of systemic antibacterial drugs & Infection & $788(84.7)$ \\
$\quad$ Intravenous antibacterial drugs & Infection & $528(56.8)$ \\
Prescription of antithrombotic drugs & Thrombosis & $542(58.3)$ \\
Prescription of antithrombotic drugs excluding heparin & Thrombosis & $207(22.3)$ \\
Prescription of systemic antifungal drugs & Infection & $374(40.2)$ \\
Intravenous antifungal drugs & Infection & $132(14.2)$ \\
Transfusion & Cytopenia & $374(40.2)$ \\
Any surgery & Any surgery & $255(27.4)$ \\
Emergency hospitalization & Emergency hospitalization & $233(25.1)$ \\
In-hospital death & In-hospital death & $203(21.8)$ \\
Prescription of antiarrhythmic drugs & Arrhythmia & $172(18.5)$ \\
Diagnosis of ischemic heart disease & Ischemic heart disease & $145(15.6)$ \\
Diagnosis of ILD & ILD & $52(5.6)$ \\
Diagnosis of intracranial hemorrhage & Intracranial hemorrhage & $24(2.6)$ \\
Stem cell transplantation & Transplantation & $15(1.6)$ \\
Endoscopic hemostasis for gastrointestinal hemorrhage & Gastrointestinal hemorrhage & $5(0.5)$ \\
Prescription of steroids for systemic use only (before treatment) & Steroid administration & 0 \\
\hline
\end{tabular}

Values are $\mathrm{n}(\%)$

*Includes in-hospital death, diagnosis of comorbidities, emergency hospitalization, surgical/diagnostic/medical procedures, and prescription of medications

ILD, interstitial lung disease

Table 5. Healthcare resource utilization in the CLL-1 and CLL-2 cohorts

\begin{tabular}{lcc}
\hline & CLL-1 & CLL-2 \\
& $\mathrm{N}=2562$ & $\mathrm{~N}=930$ \\
\hline Follow-up, days, median (Q1-Q3) & $721(363-1267)$ & $727.5(421-1193.2)$ \\
Patients requiring hospitalization, $\mathrm{n}(\%)$ & $1288(50.3)$ & $618(66.5)$ \\
Hospitalizations per patient, median (Q1-Q3) & $2(1-3)$ & $2(1-4)$ \\
Total in-hospital stay, days, median (Q1-Q3) & $29(12-65)$ & $42(17-97)$ \\
Patients requiring ER admission, n (\%) & $497(19.4)$ & $233(25.1)$ \\
ER admissions per patient per year, median (Q1-Q3) & $0.7(0.4-1.7)$ & $0.6(0.4-1.4)$ \\
Patients requiring outpatient visits, n (\%) & $2459(96.0)$ & $880(94.6)$ \\
Outpatient visits per patient per year, median (Q1-Q3) & $10.7(6.3-17.4)$ & $14.9(10.2-21.1)$ \\
\hline
\end{tabular}

CLL, chronic lymphocytic leukemia; Q1, quartile 1; Q3, quartile 3

Table 6. Healthcare claims in the CLL-1 and CLL-2 cohorts (millions of Japanese yen)

\begin{tabular}{lcc}
\hline Cost & $\begin{array}{c}\text { CLL-1 } \\
\mathrm{N}=2562\end{array}$ & $\begin{array}{c}\text { CLL-2 } \\
\mathrm{N}=930\end{array}$ \\
\hline Total cost per patient & $0.94(0.24-3.65)$ & $4.24(1.72-8.44)$ \\
Total cost per patient per year & $0.63(0.15-2.41)$ & $2.53(0.92-5.97)$ \\
Inpatient cost per patient & $1.72(0.82-3.84)$ & $2.48(1.15-5.39)$ \\
Outpatient costs per visit & $0.02(0.01-0.04)$ & $0.02(0.01-0.06)$ \\
Prescription costs per patient & $0.38(0.05-1.85)$ & $1.98(0.66-4.11)$ \\
Examination costs per patient & $0.28(0.13-0.55)$ & $0.44(0.23-0.78)$ \\
Other costs per patient & $0.26(0.03-1.40)$ & $1.33(0.40-2.98)$ \\
\hline
\end{tabular}

Values are the median (quartile 1-quartile 3)

CLL, chronic lymphocytic leukemia
2018 in Japan. It is possible that these patients received treatment at an institution not participating in the MDV database and then transferred to a participating institution where they were prescribed their 'first' registered treatment. Methotrexate was used as first-line treatment in some patients, although this is likely explained by its more common indication for rheumatism or related diseases, which cannot be elucidated from the MDV database.

Another key result of this study is that the median TIT was 1331 days. This value is more than twice as long as that reported in a US study. ${ }^{10}$ It is possible that a large number of Japanese patients were diagnosed with asymptomatic CLL during health checkups/screening and immediate treatment was not deemed necessary by the attending physician. Of patients in the CLL-1 cohort, $79.4 \%$ and $69.7 \%$ had not 
received a first-line treatment at 3 months and 1 year, respectively. In some cases, this may have been related to the "watch and wait" approach for early-stage asymptomatic CLL described in the Japanese guidelines. ${ }^{6,7}$

We also evaluated the clinical impact of CLL in terms of clinical events and associated safety risks. In the present study, systemic anti-infective drugs were prescribed to more patients than expected. We suspect that some of these prescriptions were for oral prophylactic use because the proportions of patients prescribed intravenous anti-infective drugs were lower than the overall use of systemic anti-infective drugs. Oral anti-infective drugs, such as sulfamethoxazoletrimethoprime, are commonly administered prophylactically, whereas intravenous drugs may be necessary for confirmed infections. In this study, intravenous antibacterial and antifungal drugs were administered to $56.8 \%$ and $14.2 \%$ of patients, respectively. Infections are common and a leading cause of death in patients with CLL, primarily because the treatments for CLL suppress host immunity. ${ }^{15}$ A recent trial documented grade $\geq 3$ infections in $45 \%$ of patients treated using ibrutinib at a median of 7.0 months (range 0-63.0 months) after starting treatment. ${ }^{16}$ Thus, the frequency of intravenous anti-infective drugs in our study is reasonable.

The frequency of antithrombotic drug prescriptions in the CLL-2 cohort was also higher than expected. However, this value decreased when we excluded the prescription of heparin, which is also used to maintain catheter patency. Considering the age of CLL patients, there is a high probability of cardiovascular diseases, and it may be necessary to pay attention to the risk of thrombosis and related disorders in these patients. ${ }^{17,18}$

Another objective was to understand the healthcare claims associated with the treatment of CLL in Japan. The bulk of the healthcare claims were related to the cost of drugs and hospitalization. A US study also documented considerable costs associated with the treatment of CLL, which were driven by high costs of the drugs, especially of newer drugs, and of managing AEs. ${ }^{10}$ Although the two studies should not be compared directly due to the differences in drug prices and health insurance systems, we can expect healthcare claims to increase over time in Japan with increasing use of newly approved drugs. In terms of overall costs, we should also consider the possible positive effects of newer drugs such as better disease control, fewer hospitalizations, and fewer associated severe AEs.

As an exploratory outcome, we determined the prevalence of Richter's transformation, a life-threatening complication of CLL. ${ }^{19}$ We found a higher proportion of cases of Richter's transformation in the CLL-2 cohort $(2.2 \%)$ than in the CLL-1 cohort (1.0\%), which may reflect a more advanced disease stage in the CLL-2 cohort. Another study in Japan documented transformation in two of 10 CLL patients. ${ }^{20}$ In the US, it was estimated that Richter's transformation occurs in 5-10\% of CLL patients. ${ }^{21}$ The lower rate of Richter's transformation in our study may be due to the shorter median follow-up duration (about 2 years) than in other studies.

There are some limitations to this study that require attention. First, we were unable to determine whether any of the clinical events were due to CLL itself or its treatment. Another limitation pertains to the analysis of healthcare resource utilization and costs. The resources and costs reflect the overall costs during the follow-up period and may include resources and expenses related to comorbidities. As the database does not cover every hospital in Japan, we must consider the possibility that some patients were transferred from or to another hospital not participating in the MDV database, where they received treatment (first-, second-, or third-line) for CLL or experienced other clinical events that were not recorded in the database. Accordingly, the database may underestimate these outcomes.

In conclusion, the CLIMBER-DBR study analyzed data on Japanese CLL patients within a specific period of time who were diagnosed and/or treated in a specific clinical setting. The study provides insight into the characteristics and treatment status of Japanese CLL patients, and serves as a useful benchmark for future studies in Japan. Although we found different trends in the treatment of CLL in Japan compared with those in other countries, these differences were mostly due to the approval/insurance coverage of drugs in Japan during the data collection period in this study, and the physicians probably selected the best treatment based on their understanding of their patients' conditions. As a number of new drugs have been developed and launched in recent years in Japan, more evidence, including the outcomes of these novel drugs, is required in order to improve the treatment strategy for CLL.

\section{ACKNOWLEDGMENTS}

The authors thank IQVIA for performing the analyses and Nicholas D. Smith (EMC K.K.) for medical writing support, which was funded by AstraZeneca K.K.

\section{FUNDING}

This study was funded by AstraZeneca K.K., Osaka, Japan.

\section{ETHICAL APPROVAL}

The study was approved by the institutional review board of the Clinical Research Promotion Network-Japan.

\section{AUTHOR CONTRIBUTIONS}

$\mathrm{KI}, \mathrm{JS}, \mathrm{JT}, \mathrm{KF}, \mathrm{MJ}$, and HN contributed to the study design and data analyses. MN contributed to data analyses. All authors critically reviewed the manuscript, approved the final draft, and take accountability for the accuracy and integrity of the work.

\section{DATA AVAILABILITY}

Due to the proprietary nature of the MDV database, data 
from this study cannot be shared with external researchers.

\section{CONFLICT OF INTEREST}

JT received grants and personal fees from Chugai, Kyowa Kirin, Eisai, Ono Pharmaceutical, Dainippon Sumitomo, Nippon Shinyaku, Mochida Pharmaceutical, and AstraZeneca; personal fees from Celgene, Abbvie, Takeda Pharmaceutical, Taisho Pharmaceutical, and Meiji Seika Pharma.

KI received fees for manuscript preparation from AstraZeneca; grants and personal fees from AstraZeneca; and personal fees from Takeda Pharmaceutical, Chugai, Celgene, Novartis, Abbvie, Janssen, Kyowa Kirin, Eisai, MSD, FUJIFILM Toyama Chemical, Ono Pharmaceutical, Nihon Mediphysics, Dainippon Sumitomo, Bayer, HUYA Japan, Bristol-Myers Squibb, Mundipharma, Otsuka, Daiichi Sankyo, Astellas, and Asahi Kasei Pharma.

HN received a Grant-in-Aid for Clinical Research from the National Hospital Organization; grants and personal fees from Janssen Pharmaceutical, Calgene, Mundipharma, Bayer Yakuhin, Takeda Pharmaceutical, Kyowa Kirin. Eisai, Bristol-Myers Squibb, Ono Pharmaceutical, Zenyaku Kogyo, AstraZeneca, and SymBio Pharmaceuticals; grants from Abbvie, Solasia Pharma, IQVIA Service Japan, and Nippon Shinyaku; and personal fees from Roche and Sanofi.

$\mathrm{KF}, \mathrm{MJ}$, and $\mathrm{MN}$ are employees of AstraZeneca.

JS received grants and personal fees from Chugai, Eisai, Takeda Pharmaceutical, and Celgene; grants from Kyowa Kirin, Shin-Nihon Kagaku, and Astellas, and personal fees from Janssen, Bristol-Myers Squibb, Abbvie, and AstraZeneca.

\section{REFERENCES}

1 Chihara D, Ito H, Matsuda T, et al. Differences in incidence and trends of haematological malignancies in Japan and the United States. Br J Haematol. 2014; 164 : 536-545.

2 Ministry of Health LaW. Patient survey 2017. Available at: https://www.e-stat.go.jp/stat-search/files?page=1\&layout=datalist $\&$ toukei $=00450022 \&$ tstat $=000001031167 \&$ cycle $=7 \&$ tclass $1=$ $000001124800 \&$ tclass $2=000001124803 \&$ stat_infid $=000031790842$. Accessed June 16, 2020 [in Japanese].

3 Lymphoma Study Group of Japanese Pathologists. The World Health Organization classification of malignant lymphomas in Japan: incidence of recently recognized entities. Pathol Int. 2000; $50:$ 696-702.

4 Rai KR, Jain P. Chronic lymphocytic leukemia (CLL)-Then and now. Am J Hematol. 2016; 91 : 330-340.

5 Suzumiya J, Takizawa J. Evolution in the management of chronic lymphocytic leukemia in Japan: should MRD negativity be the goal? Int J Hematol. 2020; 111 : 642-656.

6 Japanese Society of Hematology. JSH practical guidelines for hematological malignancies, 2018, Chapter I Leukemia. I Leukemia. 5 Chronic lymphocytic leukemia/small lymphocytic lymphoma (chronic lymphocytic leukemia/small lymphoctic lymphoma: CLL/SLL). Available at: http://www.jshem.or.jp/ gui-hemali/1_5.html. Accessed March 17, 2020 [in Japanese].

7 Japanese Society of Hematology. Practical Guidelines for Hematological Malignancies, 2018. Revised Version April 2020, Tokyo, Kanehara \& Co., Ltd. 2020; pp. 121-128.

8 Hallek M, Cheson BD, Catovsky D, et al. iwCLL guidelines for diagnosis, indications for treatment, response assessment, and supportive management of CLL. Blood. 2018; 131 : 2745-2760.

9 NCCN Clinical Practice Guidelines in Oncology. (NCCN Guidelines $\left.{ }^{\circledR}\right)$. Chronic Lymphocytic Leukemia/Small Lymphocytic Lymphoma. Version 4.2020_December 20, 2019. Available at: https://www.nccn.org/professionals/physician_gls/ pdf/cll.pdf. Accessed April 20, 2020.

10 Kabadi SM, Goyal RK, Nagar SP, Kaye JA, Davis KL. Treatment patterns, adverse events, and economic burden in a privately insured population of patients with chronic lymphocytic leukemia in the United States. Cancer Med. 2019; 8 : 3803-3810.

11 Burger JA, Tedeschi A, Barr PM, et al. Ibrutinib as initial therapy for patients with chronic lymphocytic leukemia. N Engl J Med. 2015; 373 : 2425-2437.

12 Mato A, Nabhan C, Kay NE, et al. Real-world clinical experience in the Connect ${ }^{\circledR}$ chronic lymphocytic leukaemia registry: a prospective cohort study of 1494 patients across 199 US centres. Br J Haematol. 2016; 175 : 892-903.

13 Mahlich J, Okamoto S, Tsubota A. Cost of illness of Japanese patients with chronic lymphocytic leukemia (CLL), and budget impact of the market introduction of ibrutinib. Pharmacoecon Open. 2017; 1 : 195-202.

14 Seymour EK, Ruterbusch JJ, Beebe-Dimmer JL, Schiffer CA. Real-world testing and treatment patterns in chronic lymphocytic leukemia: A SEER patterns of care analysis. Cancer. 2019; $125: 135-143$.

15 Tadmor T, Welslau M, Hus I. A review of the infection pathogenesis and prophylaxis recommendations in patients with chronic lymphocytic leukemia. Expert Rev Hematol. 2018; 11 : 57-70.

16 Munir T, Brown JR, O’Brien S, et al. Final analysis from RESONATE: Up to six years of follow-up on ibrutinib in patients with previously treated chronic lymphocytic leukemia or small lymphocytic lymphoma. Am J Hematol. 2019; 94 : 1353-1363.

17 Kikuchi K, Uchikado H, Morioka M, Murai Y, Tanaka E. Clinical neuroprotective drugs for treatment and prevention of stroke. Int J Mol Sci. 2012; 13 : 7739-7761.

18 Suzuki J, Shimamura M, Suda H, et al. Current therapies and investigational drugs for peripheral arterial disease. Hypertens Res. 2016; 39 : 183-191.

19 Allan JN, Furman RR. Current trends in the management of Richter's syndrome. Int J Hematol Oncol. 2019; 7 : IJH09.

20 Aoki H, Takishita M, Kosaka M, Saito S. Frequent somatic mutations in D and/or JH segments of Ig gene in Waldenstrom's macroglobulinemia and chronic lymphocytic leukemia (CLL) with Richter's syndrome but not in common CLL. Blood. 1995; $85: 1913-1919$.

21 Jain P, O'Brien S. Richter's transformation in chronic lymphocytic leukemia. Oncology (Williston Park). 2012; 26 : 1146-1152. 